08.3

\title{
Оценки скорости Ферми и эффективной массы в эпитаксиальных графене и карбине
}

\author{
() С.Ю. Давыдов
}

Физико-технический институт им. А.Ф. Иофрфе РАН,

Санкт-Петербург, Россия

E-mail: Sergei_Davydov@mail.ru

Поступило в Редакцию 2 апреля 2019г.

В окончательной редакции 2 апреля 2019г.

Принято к публикации 5 апреля 2019г.

Показано, что для слабо связанных с подложкой бесщелевых графена и карбина-кумулена естественной характеристикой является скорость Ферми электрона, тогда как при наличии щели (графен со щелью и карбин-полиин) такой характеристикой является эффективная масса электрона. Приведены соответствующие аналитические оценки.

Ключевые слова: закон дисперсии, плотность состояний, металлическая и полупроводниковая подложки.

DOI: 10.21883/PJTF.2019.13.47950.17819

Хорошо известно [1], что в приближении сильной связи с учетом взаимодействия только ближайших соседей линеаризованный закон дисперсии электронов в однослойном графене имеет вид

$$
\omega_{G}(\mathbf{q})=\varepsilon_{\mathrm{D}} \pm \hbar v_{\mathrm{F}}|\mathbf{q}|
$$

(низкоэнергетическое приближение). Здесь $\varepsilon_{\mathrm{D}}-$ энергия точки Дирака, $\hbar-$ приведенная постоянная Планка, $v_{\mathrm{F}}=3 a t / 2 \hbar \sim 10^{6} \mathrm{~m} / \mathrm{s}-$ скорость Ферми, $t \sim 3 \mathrm{eV}$ - энергия перехода электрона между ближайшими соседями, расстояние между которыми равно $a, \mathbf{q}=\mathbf{K}-\mathbf{k}$ - волновой вектор, отсчитываемый от волнового вектора Дирака $\mathbf{K}=a^{-1}(2 \pi / 3 \sqrt{3}, 2 \pi / 3)$, положительная (отрицательная) ветвь спектра соответствует зоне проводимости (валентной зоне). Такому спектру отвечает нулевая эффективная масса носителей, a его характеристикой является фермиевская скорость $v_{\mathrm{F}}$. Соответствующую закону дисперсии (1) плотность состояний (в расчете на один адатом) можно представить в виде

$$
\rho_{G}(\omega)=\frac{3 \sqrt{3} a^{2}}{4 \pi \hbar^{2} v_{\mathrm{F}}^{2}}\left|\omega-\varepsilon_{\mathrm{D}}\right|,
$$

где $\omega-$ энергетическая переменная (см. [1]). Отметим, что формула (2) справедлива для области энергий $\left|\omega-\varepsilon_{\mathrm{D}}\right| \ll t$.

Выражения (1) и (2) отвечают свободному (не связанному с подложкой) листу графена. Учету влияния взаимодействия графен-подложка на фермиевскую скорость в эпитаксиальном графене (эпиграфене) посвящены работы [2,3]. В [2] показано, что фермиевская скорость электрона в эпиграфене $\tilde{v}_{\text {F }}$ определяется выражением

$$
\tilde{v}_{\mathrm{F}} / v_{\mathrm{F}}=F\left(\varepsilon_{\mathrm{D}}\right), \quad F(\omega)=(1-d \Lambda(\omega) / d \omega)^{-1 D},
$$

где $\Lambda(\omega)$ - функция сдвига энергетических уровней изолированного графена, вызванного взаимодействием с подложкой (здесь и далее тильда относится к характеристикам эпитаксиальных структур). Подчеркнем, что в [2,3] рассматривался бесщелевой эпиграфен, т.е. считалось, в частности, что подложка не наводит в графеновом монослое зону запрещенных состояний. Последнее условие выполняется для квазисвободного, т.е. слабо связанного с подложкой листа графена, когда $V^{2} / W^{2} \ll 1$, где $V-$ матричный элемент связи графен-подложка, $W$ - характерная ширина зон разрешенных состояний подложки. Если рассматривать металлическую подложку, то в простейшем приближении ее плотность состояний $\rho_{m}(\omega)$ можно считать постоянной (модель широкой зоны Андерсона [4]), откуда получим $\Lambda(\omega)=0$ и $F(\omega)=1$. Более сложные модели, рассмотренные в $[2,3]$, приводят к неравенству $F(\omega)>1$.

Для полупроводниковой подложки, как и в [3], положим плотность состояний равной $\rho_{s c}(\omega)=A_{s c} \sqrt{|\omega|-E_{g} / 2}$ при $|\omega| \geqslant E_{g} / 2$ и $\rho_{s c}(\omega)=0$ при $|\omega|<E_{g} / 2$, где $E_{g}-$ ширина запрещенной зоны, $A_{s c}-$ коэффициент с размерностью $\mathrm{eV}^{-3 / 2}$, нуль энергии помещен в центр запрещенной зоны (модель Перссона-Линдефельта для $4 H$ - и $6 H$-политипов карбида кремния $[5,6])$. В области запрещенной зоны $|\omega|<E_{g} / 2$ функция сдвига $\quad \Lambda_{s c}(\omega)=A_{s c} V_{s c}^{2}\left(f_{-}(\omega)-f_{+}(\omega)\right), \quad$ где $f_{ \pm}(\omega)=\pi \sqrt{\mp \omega+E_{g} / 2}, \quad V_{s c}-$ матричный элемент взаимодействия графен-подложка [3]. Тогда для функции $F_{s c}\left(\varepsilon_{\mathrm{D}}\right)$ получим выражение

$$
\begin{aligned}
F_{s c}\left(\varepsilon_{\mathrm{D}}\right) & =\left(1+\frac{\pi A_{s c} V_{s c}^{2}}{2}\left(\frac{1}{\sqrt{\left(E_{g} / 2\right)-\varepsilon_{\mathrm{D}}}}\right.\right. \\
& \left.\left.+\frac{1}{\sqrt{\left(E_{g} / 2\right)+\varepsilon_{\mathrm{D}}}}\right)\right)^{-1} .
\end{aligned}
$$


Отсюда следует, что функция $F_{s c}\left(\varepsilon_{\mathrm{D}}\right)<1$ и существенно зависит от $\varepsilon_{\mathrm{D}}$, обращаясь в нуль при $\left|\varepsilon_{\mathrm{D}}\right|=E_{g} / 2$ и достигая максимума

$$
F_{s c}^{0}=\left(1+\pi A_{s c} V_{s c}^{2} / \sqrt{E_{g} / 2}\right)^{-1}
$$

при $\varepsilon_{\mathrm{D}}=0$ (приведенная в [3] формула (10) ошибочна и ее следует заменить выражением (4)). Такое поведение функции $F_{s c}\left(\varepsilon_{\mathrm{D}}\right)$ качественно совпадает с результатами, полученными в [2] в рамках ступенеобразной плотности состояний Халдейна-Андерсона [4], хотя модель Перссона-Линдефельта [5] скачков плотности состояний не содержит (см. обсуждение причин артефактного обращения в нуль фермиевской скорости на границах запрещенной зоны в [2]).

Заменяя в (2) $v_{\mathrm{F}}$ на $\tilde{v}_{\mathrm{F}}$ и $\varepsilon_{\mathrm{D}}$ на перенормированную (в линейном приближении) энергию точки Дирака $\tilde{\varepsilon}_{\mathrm{D}} \approx \varepsilon_{\mathrm{D}}+\Lambda_{s c}\left(\varepsilon_{\mathrm{D}}\right)$, легко показать, что число заполнения адатома углерода квазисвободного эпиграфена равно

$$
\tilde{n}_{G} \approx 1 \pm \delta \tilde{n}, \quad \delta \tilde{n}=\frac{\left(\varepsilon_{\mathrm{F}}-\tilde{\varepsilon}_{\mathrm{D}}\right)^{2}}{\pi \sqrt{3}\left(F_{s c}^{0}\right)^{2} t^{2}},
$$

где $\varepsilon_{\mathrm{F}}-$ энергия фермиевского уровня, знак плюс соответствует $\varepsilon_{\mathrm{F}}>\tilde{\varepsilon}_{\mathrm{D}}$, знак минус отвечает $\varepsilon_{\mathrm{F}}<\tilde{\varepsilon}_{\mathrm{D}}$. Концентрация носителей равна $\tilde{N}_{c}=\delta \tilde{n} / S_{1}$, где $S_{1}=3 \sqrt{3} a^{2} / 4-$ площадь, приходящаяся на один адатом графена.

Сделаем некоторые численные оценки. Положим $V_{s c}=1 \mathrm{eV} \quad$ и учтем, что для подложки $6 H$-SiC $A_{s c}=0.2 \mathrm{eV}^{-3 / 2}$ [6] и $E_{g}=3 \mathrm{eV}$ [7]. Поскольку сродство к электрону $\chi=3.45 \mathrm{eV}$ [7] и работа выхода свободного графена $\phi=4.5 \mathrm{eV}$ [8], получим $\varepsilon_{\mathrm{D}}=0.45 \mathrm{eV}$ и $\tilde{\varepsilon}_{\mathrm{D}}=0.38 \mathrm{eV}$. Тогда получаем $F_{s c}=0.66$. Если подложка $\mathrm{SiC}$ обладает собственной проводимостью $\left(\varepsilon_{\mathrm{F}}=0\right)$, то при $t=3 \mathrm{eV}$ находим $\delta \tilde{n} \approx 6.8 \cdot 10^{-3}$ и $\tilde{N}_{c}=p \approx 2.6 \cdot 10^{13} \mathrm{~cm}^{-2}$. Вновь полагая $V_{s c}=1 \mathrm{eV}$, в случае подложки $4 H-\mathrm{SiC}\left(E_{g}=3.23 \mathrm{eV}, \chi=3.17 \mathrm{eV}\right.$ [7]) имеем $\varepsilon_{D}=0.29 \mathrm{eV}$ и $\tilde{\varepsilon}_{\mathrm{D}}=0.24 \mathrm{eV}$. Тогда $F_{s c}=0.67$, и при $\varepsilon_{\mathrm{F}}=0, t=3 \mathrm{eV}$ получаем $\delta \tilde{n} \approx 2.6 \cdot 10^{-3}$ и $\tilde{N}_{c}=p \approx 1.0 \cdot 10^{13} \mathrm{~cm}^{-2}$ ( $p$ - концентрация дырок $)$.

Если каким-либо образом (деформацией, допированием, адсорбцией) в свободном от подложки идеальном листе графена наведена щель шириной $2 \Delta$, то закон дисперсии приобретает вид

$$
\omega_{G}^{\prime}(\mathbf{q})=\varepsilon_{\mathrm{D}} \pm \sqrt{\Delta^{2}+\left(\hbar v_{\mathrm{F}} q\right)^{2}} .
$$

Характеристикой такого спектра является уже не обращающаяся в нуль фермиевская скорость $v_{\mathrm{F}}$, а эффективная масса $m^{*}=\hbar^{2}\left[\partial^{2}\left|\omega_{\mathrm{GLC}}(q)\right| / \partial q^{2}\right]_{q=0}^{-1}$, равная $\quad\left(m^{*}\right)^{-1}=m_{e, h}^{-1}= \pm v_{\mathrm{F}}^{2} / \Delta$. Легко показать, что при наличии полупроводниковой подложки получим $m^{*} / \tilde{m}^{*}=F_{s c}\left(\varepsilon_{\mathrm{D}} \pm \Delta\right)$, где верхний знак соответствует электронам, нижний - дыркам. Здесь, как и выше, рассматривается квазисвободный графен.
Перейдем теперь к одномерным углеродным структурам, или карбинам $[9,10]$. Различают два вида карбинов: металлический кумулен с двойными связями $(\cdots=\mathrm{C}=\mathrm{C}=\ldots)$ и полупроводниковый полиин с чередующимися одинарными и тройными связями $(\cdots \equiv \mathrm{C}-\mathrm{C} \equiv \mathrm{C}-\mathrm{C} \equiv \ldots)$. Согласно $[11]$, для полиина закон дисперсии имеет вид

$$
\begin{gathered}
\omega_{p o l}^{ \pm}(k)=\varepsilon_{\mathrm{D}} \pm R(k), \\
R(k)=2 t^{\prime} \sqrt{A_{1}^{2}(k)+A_{2}^{2}(k)}, \quad\left|k_{p o l}\right| \leqslant \pi / 2 a,
\end{gathered}
$$

где

$$
\begin{aligned}
& A_{1}(k)=\cos (\alpha k a) \cos (k a)-\tau \sin (\alpha k a) \sin (k a), \\
& A_{2}(k)=\sin (\alpha k a) \cos (k a)+\tau \cos (\alpha k a) \sin (k a),
\end{aligned}
$$

$\alpha=\Delta a / 2 a$ и $\tau=\Delta t / 2 t^{\prime}$. Здесь учтено, что одинарная связь $\mathrm{C}-\mathrm{C}$ имеет длину $a_{1}=1.265 \AA$, тройная связь $\mathrm{C} \equiv \mathrm{C}-$ длину $a_{2}=1.301 \AA$ [12]. Соответствующие энергии перехода $t_{1,2}=\left|\eta_{p p \pi}\right|\left(\hbar^{2} / m_{0} a_{1,2}^{2}\right)$, вычисленные по методу Харрисона [13] $\left(\eta_{p р \pi}=-0.63\right)$, равны $t_{1}=3.00 \mathrm{eV}$ и $t_{2}=2.84 \mathrm{eV}$. Полагая $\Delta a=a_{1}-a_{2}$, $2 a=a_{1}+a_{2}, \quad \Delta t=t_{1}-t_{2}, \quad 2 t^{\prime}=t_{1}+t_{2}, \quad$ получим $\Delta a / a \approx-0.03$ и $\Delta t / 2 t^{\prime} \approx 0.03$. Согласно (8), у полиина при $|k|=\pi / 2 a$ в электронном спектре имеется щель $\Delta_{p o l}=2 R(\pi / 2 a)=2 \Delta t \approx 0.32 \mathrm{eV}$. Разложив функции $A_{1,2}(k)$ до второго порядка по $q=(\pi / 2 a)-k$ и считая $\alpha=\Delta a / 2 a, \tau=\Delta t / 2 t^{\prime}$ малыми параметрами, получим $R(k) \approx \Delta t \sqrt{1+(q a / \tau)^{2}}$ и $m^{*} / m_{0}=\hbar^{2} \tau^{2} / m_{0} a^{2} \Delta t \approx 0.03$.

Для кумулена, рассматриваемого как обычная одноатомная цепочка, имеет место закон дисперсии вида

$$
\omega_{\text {cum }}(k)=\varepsilon_{\mathrm{D}}-2 t \cos (k a),\left|k_{\text {cum }}\right| \leqslant \pi / a,
$$

где $a=1.282 \AA$ [12], так что в рамках теории Харрисона [13] получаем $t=2.92 \mathrm{eV}$ и $m_{\text {сит }}=\hbar^{2} / 2 t m_{0} a^{2} \approx 0.79$. Таким образом, налицо явное несоответствие: малая деформация кумулена, превращающая его в полиин, в 26 раз увеличивает эффективную массу электрона. Предположим поэтому, что соседние атомы углеродной цепочки сдвинуты по оси $y$ относительно друг друга на бесконечно малые расстояния $\pm 0^{+}$. При этом цепочка вследствие ее пилообразности (хотя и исчезающе малой) становится двухатомной, в результате чего зона Бриллюэна сжимается в 2 раза, так что $\left|k_{\text {cum }}\right| \leqslant \pi / 2 a$. Тогда получаем $v_{\mathrm{F}}=t a / \hbar \approx 0.6 \cdot 10^{6} \mathrm{~m} / \mathrm{s}$ и $m_{\text {cum }}=0$. В рамках такой модели кумулен является неустойчивой структурой и должен испытать пайерлсовский переход в полиин. Легко показать, что для эпикарбина на полупроводниковой подложке выполняются соотношения, аналогичные соотношениям для эпиграфена:

$$
\tilde{v}_{\mathrm{F}} / v_{\mathrm{F}}=F_{s c}\left(\varepsilon_{\mathrm{D}}\right) \text { и } m^{*} / \tilde{m}^{*}=F_{s c}\left(\varepsilon_{\mathrm{D}} \pm \Delta t\right) .
$$

Итак, в настоящей работе мы рассмотрели непосредственное влияние взаимодействия углеродных структур с полупроводниковой подложкой на их транспортные 
характеристики. Показано, что такой субстрат понижает фермиевскую скорость электрона в бесщелевой структуре и увеличивает его эффективную массу в структуре со щелью. Рассмотрение велось в рамках одночастичного приближения. Отметим, что учету влияния кулоновского взаимодействия электронов в графене на скорость Ферми посвящен ряд публикаций (см., например, работы [14-17] и ссылки в них). Показано, в частности, что такое взаимодействие может менять фермиевскую скорость в разы. При этом, однако, полупроводниковая подложка рассматривалась только как 1) источник электронов (дырок) в графене и 2) экранирующая кулоновское отталкивание диэлектрическая среда, тогда как роль взаимодействия графен-субстрат полностью игнорировалась. В дальнейшем мы планируем учесть одновременно все эти факторы, следуя для учета корреляции результатам работ $[11,18]$ и включив в рассмотрение дополнительно кулоновское отталкивание электронов графена и подложки.

\section{Список литературы}

[1] Castro Neto A.H., Guinea F., Peres N.M.R., Novoselov K.S., Geim A.K. // Rev. Mod. Phys. 2009. V. 81. N 1. P. 109-162.

[2] Алисултанов 3.3. // Письма в ЖТФ. 2013. Т. 39. В. 13. C. 32-39.

[3] Давыдов С.Ю. // ФТТ. 2014. Т. 56. В. 4. С. 816-820.

[4] Давыдов С.Ю., Лебедев А.А., Посредник О.В. Элементарное введение в теорию наносистем. СПб.: Лань, 2014. $192 \mathrm{c}$.

[5] Persson C., Lindefelt U. // Mater. Sci. Forum. 1998. V. 264268. P. 275-278.

[6] Давыдов С.Ю. // ФТП. 2014. Т. 48. В. 1. С. 49-54.

[7] Давыдов С.Ю. // ФТП. 2007. Т. 41. В. 6. С. 718-720.

[8] Kim J.-H., Hwang J.H., Suh J., Tongay S., Kwon S., Hwang C.C., Wu J., Park J.Y. // Appl. Phys. Lett. 2013. V. 103. N 17. P. 171604.

[9] Banhart F. // J. Nanotechnol. 2015. V. 6. P. 559-569.

[10] Shi L., Rohringer P., Wanko M., Rubio A., Waserroth S., Reich S., Cambre S., Wenseleers W., Ayala P., Pichler T. // Phys. Rev. Mater. 2017. V. 1. N 7. P. 075601; arXiv: 1705.02259

[11] Давыдов С.Ю. // ФТП. 2019. Т. 53. В. 7. С. 971-977.

[12] Tongay S., Senger R.T., Dag S., Ciraci S. // Phys. Rev. Lett. 2004. V. 93. N 13. P. 136404.

[13] Harrison W.A. // Phys. Rev. B. 1983. V. 27. N 6. P. 3592 3604.

[14] Elias D.C., Gorbachev R.V., Mayorov A.S., Morozov S.V., Zhukov A.A., Blake P., Ponomarenko L.A., Grigorieva I.V., Novoselov K.S., Guinea F., Geim A.K. // Nature Phys. 2011. V. 7. N 9. P. 701-704.

[15] Hwang C., Siegel D.A., Mo S.-K., Regan W., Ismach A., Zhang Y., Zettl A., Lanzara A. // Sci. Rep. 2012. V. 2. P. 590593.

[16] Siegel D.A., Regan W., Fedorov A.V., Zettl A., Lanzara A. // Phys. Rev. Lett. 2013. V. 110. N 14. P. 146802.

[17] Stauber T., Parida P., Trushin M., Ulybyshev M.V., Boyda D.L., Schliemann J. // Phys. Rev. Lett. 2017. V. 118. N 26. P. 266801.

[18] Давыдов С.Ю. // ФТТ. 2017. Т. 59. В. 8. С. 1650-1658. 